\title{
Radiation Transfer in Gamma-Ray Bursts
}

\section{B. J. Carrigan*† and J. I. Katz*}

\begin{abstract}
We have calculated gamma-ray radiative transport in regions of high energy density, such as gamma-ray burst source regions, using a discrete ordinate, discrete energy group method. The calculations include two-photon pair production and annihilation, as well as three-photon pair annihilation. The radiation field itself acts as an absorbing medium, and the optical depth depends on its intensity, so the problem is intrinsically nonlinear. Spherical divergence produces effective collimation of the flux. At high optical depth the high energy $(E>1 \mathrm{MeV})$ portion of the emergent spectrum assumes a nearly universal form. An approximate limit is derived for the high energy flux from a gamma-ray burst source region of given size, and the implications of this limit for the distance to the March 5, 1979 event are briefly discussed. We discuss more generally the problem of very luminous bursts, and implications of Galactic halo distances for flare models.
\end{abstract}

Subject headings: Gamma Rays: Bursts, Radiative Transfer

* Department of Physics and McDonnell Center for the Space Sciences, Washington U., St. Louis, Mo. 63130

$\dagger$ Present Address: Department of Physics, Millikin U., Decatur, Ill. 62522 


\section{INTRODUCTION}

Gamma-ray bursts pose an unusual and interesting problem in radiation transport. In regions of low matter density, but high gamma-ray radiation density, the chief source of opacity at gamma-ray $\left(>\frac{1}{2} \mathrm{MeV}\right)$ energy is pair production by interaction with other gamma-rays. The opacity is proportional to the radiation energy density, making the radiation transport equation intrinsically nonlinear. Because of the kinematics of twophoton pair production, the opacity is also a sensitive function of the angular and spectral distribution of the radiation field.

The purpose of this paper is to describe the results of gamma-ray radiation transport calculations under the influence of pair-production opacity, making the approximation that other sources of opacity (such as Compton scattering) are negligible. A preliminary account of our work, restricted to the case of slab-symmetry, was reported by Carrigan and Katz (1984). Because of the importance of the photon angular distribution to the opacity, spherical divergence is essential to a realistic calculation, and it is used in the calculations reported here.

These radiation transport calculations are also of interest because arguments based on pair-production opacities have been used to constrain the distances of gamma-ray burst sources. Direct estimates of their distances are controversial, and range from $<100 \mathrm{pc}$ to cosmological values. There are no universally accepted identifications of burst sources with quiescent counterparts whose distances might be determined by independent astronomical arguments. Statistical arguments, using empirical $\log N / \log S$ relations, have a long history of controversy, because of questions of completeness of sampling at lower flux levels. This controversy may be resolved, at least for the more common classes of burst sources, by data from the BATSE on GRO, but some of the most interesting sources, such as the soft gamma repeaters (SGR), are few enough to preclude statistical analysis. The SGR also have a large range of intrinsic brightness, complicating $\log N / \log S$ analysis, but 
excluding models (such as thermonuclear) which predict a "standard candle" of luminosity.

Perhaps the most interesting problem is presented by GBS 0526-66 (the March 5, 1979, source; Cline 1980), whose position on the sky coincides with the supernova remnant N 49. However, N 49 lies in the Large Magellanic Cloud, so the inferred luminosity of bursts from this source is huge, and many scientists have been reluctant to accept the identification of N 49 with GBS 0526-66. Faced with this lack of information, attempts have been made to obtain an upper bound on burster distances from the observed burst spectra.

The argument (Schmidt 1978, Cavallo and Rees 1978, Katz 1982, Epstein 1985) proceeds as follows: two photons which are above the threshold for electron-positron pair production

$$
E_{1} E_{2}\left(1-\cos \theta_{12}\right) \geq 2\left(m_{e} c^{2}\right)^{2}
$$

may produce a pair, where $\theta_{12}$ is the angle between the directions of the two gammarays. The cross section for pair production reaches a maximum at a finite CM photon energy (roughly $700 \mathrm{KeV}$ ); if the photon spectrum is not sharply peaked high-energy photons will, therefore, form pairs predominantly with low-energy photons. Because any reasonable source spectrum will contain many more low- or moderate-energy photons than high-energy photons, the emergent spectrum will differ most markedly from the source spectrum at high photon energies $(E>1 \mathrm{MeV})$, at which it will be heavily depleted. The electron-positron pairs eventually annihilate to produce two (infrequently three) photons, but usually not one high- and one low-energy photon. The result is that high-energy photons are preferentially removed from the spectrum. The observation of a measurable amount of flux with $E>1 \mathrm{MeV}$ is thus not expected unless the optical depth to pair production is unity or less. However, the optical depth is given approximately by

$$
\tau \sim n_{\gamma} r_{e}^{2} R
$$

where $n_{\gamma}$ is the number density, at the source, of photons with energy above threshold for 
pair production off the observed high-energy photons, $r_{e}$ is the classical electron radius $e^{2} /\left(m_{e} c^{2}\right)$ (the cross sections for pair production and annihilation, as well as for Compton scattering, are all $\sim r_{e}^{2}$ at these semi-relativistic energies), and $R$ is the radius of the source. The condition of low $(\tau<1)$ optical depth then yields

$$
n_{\gamma}<r_{e}^{-2} R^{-1}
$$

or, for the flux at the source,

$$
F_{0} \sim n_{\gamma} c<c r_{e}^{-2} R^{-1}
$$

The flux at the solar system, a distance $d$ from the source, is

$$
F(d)=F_{0}(R / d)^{2}<c R d^{-2} r_{e}^{-2},
$$

and hence

$$
d<\left[c R F(d)^{-1} r_{e}^{-2}\right]^{1 / 2},
$$

or

$$
d<200 \mathrm{pc}(R / 10 \mathrm{~km})^{1 / 2}\left[F(d) / 1 \mathrm{~cm}^{-2} \mathrm{~s}^{-1}\right]^{-1 / 2} .
$$

For a typical burst, the peak flux implies an upper bound to the source distance of a few hundred to a few thousand parsecs. For the intense March 5, 1979 event, however, the distance limit inferred in this way from the peak flux is $\sim 30 \mathrm{pc}$; in addition, the presence of an emission feature in the initial 4-second spectrum of this burst implies a tighter bound of $\sim 7 \mathrm{pc}$ (based on a Comptonization argument); even tighter bounds of $4.3 \mathrm{pc}$ and $<1 \mathrm{pc}$ (Helfand and Long 1979, Katz 1982) have been proposed. The reality of apparent emission features in burst spectra has been questioned, however (Fenimore, et al. 1982; Nolan, et al. 1984; Teegarden 1984; Liang and Petrosian 1986) so the tightest proposed distance bounds ( $<10$ pc) may be discounted. The looser bounds, based simply on the existence of a high-energy continuum, will be the only distance bound considered here. In view of the tentative localization of the 5 March 1979 source in the LMC, inconsistent by orders 
of magnitude with even the loosest of the bounds mentioned above, it is of interest to investigate any loopholes in the distance bounds.

One possible loophole is that the bounds given above are approximate; a more precise calculation of the cross sections may provide an optical depth at much larger (or smaller) fluxes at the source. A consideration of the exact pair-production cross section, and a number of reasonable source spectra, leads to a higher upper bound than the crude estimate given above. For the March 5, 1979 source, the high-energy continuum implies an upper bound of about 150 pc (Epstein 1985). Though this is a five-fold increase in the distance bound, it is still far too small to permit the March 5, 1979 source to be extragalactic.

A second loophole exists if the source produces a strongly collimated beam of photons. In this case, even high energy photons are below threshold for pair production, because $\theta_{12}$ is small. A collimated relativistically-outflowing gas of electrons and positrons would, on general kinematic grounds, be expected to produce outward collimated radiation. Such collimated outflows are inferred in superluminally expanding active galactic nuclei, and might be expected following any release of energy density $\gg \rho c^{2}$, where $\rho$ is the local rest mass density, but their detailed mechanisms are obscure.

A final possible loophole begins with a consideration of all the reactions a system of photons and charged particles can undergo. These processes include Compton scattering, bremsstrahlung, and three- (or more) photon annihilation. In addition, the presence of a magnetic field would allow other processes, such as synchrotron radiation and one-photon pair production and annihilation. Most of these processes are dissipative, in the sense that the number of particles increase, so energy is distributed among more particles at the end of the reaction than at the beginning. On the other hand, high-energy photons are also produced. As a result, the high-energy flux may be augmented as well as depleted by interactions, and this may serve to weaken the distance bounds given above; in other words, a significant high energy flux may emerge from a region which is optically thick to high energy photons. 
There are four ways in which a very intense source of gamma-rays might evade the bound (6):

1. Diffusion of radiation through optically thick regions. However, because of the proportionality of effective volume opacity to the radiation energy density, the required increase of energy density with distance into the opaque region is exponential (as seen in a simple model calculation; Katz 1982), rather than linear (the usual result for linear radiation transport).

2. Repeated pair production and annihilation will, through the occasional three-body process, lead to a softening of the spectrum.

3. The presence of a "window" in the opacity for collimated photons suggests that in a region opaque to pair production much of the radiation may emerge through this window, in analogy to the great contribution of windows in the material opacity to radiation flow in the usual (Rosseland mean) approximation.

4. Outward flow in spherical geometry leads to a purely geometrical radial collimation of the photon flux (any ray approaches the radial direction as $r \rightarrow \infty$ ), increasing the pair-production threshold.

In order to investigate these processes and to determine how valid the flux-derived distance bounds are, one must solve the transport equations in a self-interacting photon system, including the effects of spherical divergence. Previous calculations (Carrigan and Katz 1984, 1987; Guilbert and Stepney 1985) assumed slab-symmetry. In $\S I I$ we discuss the equations of radiation transport, and in $§ I I I$ numerical methods for their solution. $\S I V$ contains the results of our calculations. In $\S \mathrm{V}$ we present a summary discussion and conclusions.

\section{RADIATIVE TRANSPORT}

The transport equation for a field $\psi$, neglecting scattering, is

$$
\frac{\partial \psi}{\partial t}+v \hat{\Omega} \cdot \nabla \psi=S-A \psi,
$$


where $\hat{\Omega}$ is the unit direction vector, $S$ (the emission term) is a source of particles (photons, electrons, or positrons), and $A$ is the absorption coefficient. In many problems, $S$ and $A$ are independent of the field, $\psi$. The transport equation would then be linear, although $S$ and $A$ often depend on $\psi$ indirectly, through the effects of $\psi$ on other quantities, such as the temperature in LTE radiative transport and level occupation densities in non-LTE transport. The drift velocity $v$ is very different for photons, which stream freely at the speed of light between interactions, than for charged particles which may be confined by a magnetic field.

In the present case, the absorption of photons arises from photon-photon interactions, while the absorption of charged particles is due to electron-positron annihilation. As a result, the absorption coefficients are proportional to the fields themselves, so the absorption terms are quadratic in the fields:

$$
\begin{aligned}
A_{\gamma}(E, \hat{\Omega}) & =\int \psi_{\gamma}\left(E^{\prime}, \hat{\Omega}^{\prime}\right) Q\left(E, E^{\prime}, \hat{\Omega}, \hat{\Omega}^{\prime}\right) d E^{\prime} d \hat{\Omega}^{\prime}, \\
A_{e}(E) & =\int \psi_{e}\left(E^{\prime}\right) P\left(E, E^{\prime}\right) d E^{\prime},
\end{aligned}
$$

where $Q$ and $P$ are known functions and $A_{\gamma}, A_{e}, \psi_{\gamma}$, and $\psi_{e}$ implicitly depend on space and time. In principle, $A_{e}(E), \psi_{e}\left(E^{\prime}\right)$, and $P\left(E, E^{\prime}\right)$ also depend on angle, but this dependence is not sharp. Charged particle distributions may be isotropized by Coulomb scattering, and their pair-production source is not highly collimated. Cyclotron radiation appears to anisotropize electron distributions rapidly, but when emission is rapid so is absorption, with a cancelling effect, and the net anisotropization rate may not be large. Because these effects are difficult to calculate quantitatively we approximate the charged particle distributions as isotropic, so that $A_{e}, \psi_{e}$, and $P$ also implicitly include integrals over their isotropic angular distributions. 
The transport equations are unchanged by the following scale transformation:

$$
\begin{aligned}
r & \rightarrow \lambda^{-1} r \\
t & \rightarrow \lambda^{-1} t \\
I \equiv \psi_{\gamma} & \rightarrow \lambda I \\
n_{e} \equiv \psi_{e} & \rightarrow \lambda n_{e} \\
S & \rightarrow \lambda^{2} S
\end{aligned}
$$

A solution of the transport equations for conditions characteristic of neutron stars may therefore be scaled to describe transport in other objects, such as active galactic nuclei and other gamma-ray sources (Matteson 1983, Carrigan and Katz 1987).

The presence of photon-photon pair production makes the transport problem nonlinear. One consequence of this is that we cannot specify the optical depth in advance; it must be calculated from the solution to the equations. As a result, the optical depth is not a particularly useful parameter in this problem. Instead, we define $\beta$, which characterizes a family of solutions (all related by the scale transformation [10]):

$$
\beta \equiv \frac{r_{e}^{2}}{R c} \int_{m_{e} c^{2}}^{\infty} d E \int_{R}^{\infty} d r r^{2} S(E, r) ;
$$

$S$ is the source spectrum (the spectrum produced by the primary radiation process which is assumed to supply the burst's energy), $E$ the photon energy, and $r$ the radius. The source region has been assumed to be the range $R \leq r<\infty$, where $R$ might be the neutron star's radius. A low-energy cut-off in the definition of $\beta$ is required for many reasonable model source spectra (e.g., a power-law) to keep the power finite; a natural cut-off is $511 \mathrm{keV}$ because lower energy photons are less effective pair-producers, and we adopted it in (11). In addition, for a source spectrum which does not vanish sufficiently rapidly at infinity, a high-energy cut-off is similarly needed. In the calculations reported here, a high-energy cut-off of $2555 \mathrm{keV}$ was used where such a cut-off was required. In contrast to the optical depth, $\beta$ may be specified at the start of a transport calculation, and is therefore a useful 
parameter of the problem. For small $\beta$, the radiation field and optical depth are both proportional to $\beta$.

In spherically symmetric geometry, the transport equation for an azimuthally symmetric radiant intensity $I(r, t, E, \mu)$ may be written as

$$
\frac{\partial I}{\partial t}+c\left(\frac{\mu}{r^{2}} \frac{\partial\left(r^{2} I\right)}{\partial r}+\frac{1}{r} \frac{\partial\left[\left(1-\mu^{2}\right) I\right]}{\partial \mu}\right)=G\left[r, t, E, \mu, I, n_{e}\right],
$$

where $\mu$ is the cosine of the angle between the photon's direction and an outward radial vector. The left hand side of (12a) represents the purely geometrical effects of free-streaming photons. $G$ includes source and absorption terms:

$G\left[r, t, E, \mu, I, n_{e}\right]=n_{e}^{2} \epsilon(T, E)-I(r, t, E, \mu) \int I\left(r, t, E^{\prime}, \mu^{\prime}\right) Q\left(E, E^{\prime}, \mu, \mu^{\prime}\right) d E^{\prime} d \mu^{\prime}+S(E, r)$.

The annihilation term is approximated

$$
\begin{aligned}
\epsilon(E, T) & =\epsilon_{2 \gamma}(E, T)+\epsilon_{3 \gamma}(E, T) \\
& \approx \epsilon_{2 \gamma}(E, T)+\alpha \epsilon_{2 \gamma}\left(\frac{3}{2} E, T\right),
\end{aligned}
$$

where $\epsilon_{2 \gamma}$ is the thermally averaged annihilation spectrum calculated by Svensson (1983), and the much more complex (and not readily available) thermally averaged three-photon contribution is roughly approximated by the scaled two-photon spectrum, as indicated, with $\alpha \approx 1 / 137$ being the usual fine structure constant. Because three-photon annihilation is small and important only when each photon undergoes many $(>1 / \alpha)$ pair production and annihilation cycles this rough approximation is adequate; its significance is that it increases the number of photons and particles in the system, degrading their mean energy, and its quantitative value and form are comparatively unimportant. Similarly, neglecting Compton scattering affects lepton-photon energy exchange, both directly by Compton recoil and indirectly by increasing the effective optical depth and thereby increasing the effects of other physical processes.

Charged particles are generated only by pair production. The electrons and positrons were assumed to form identical (isotropic) relativistic Maxwell-Boltzmann distributions; 
therefore, a number density and temperature (both functions of $r$ and $t$ ) suffice to characterize the charged-particle subsystem. Isotropy is an approximation, but because the charged particle source is not collimated, and annihilation rates at our energies are not strongly angle-dependent, its use is justified. We assume that the mean charged-particle velocity is zero and ignore their spatial transport. This is justified if they are trapped by a strong magnetic field, as is likely near a neutron star (flare-like models of gammaray bursts require high magnetic fields; see Katz 1982). The resulting equation for the charged-particle density is

$$
\frac{\partial n_{e}}{\partial t}=\int I(E, \mu) I\left(E^{\prime}, \mu^{\prime}\right) Q\left(E, E^{\prime}, \mu, \mu^{\prime}\right) d E d E^{\prime} d \mu d \mu^{\prime}-n_{e}^{2} W(T),
$$

where $Q$ is the azimuthally averaged pair-production coefficient and $W$ is the thermallyaveraged annihilation coefficient; we assume zero baryon density so that the electron and positron densities are each $n_{e}$. The matter temperature $T$ is determined from the mean energy per charged particle, which is found from the conservation of energy, using the mean energy of the pairs produced and of the pairs annihilated. Additional details are presented by Carrigan (1987).

Because our purpose was to study nonlinear radiative transport as cleanly as possible, we only considered pair production and two- and three-photon pair annihilation. Compton scattering would destroy any spectral lines (for optical depths greater than unity) and tend to thermalize the emergent spectrum. However, the model source spectra considered lacked spectral lines, and the processes considered also act to thermalize the spectra; therefore, Compton scattering would not be likely to make a qualitative difference. The inclusion of a spectral softening process (three-photon pair annihilation) is important; in the absence of such a process, the mean particle energy could not change. There are many softening processes; again, the precise one or ones chosen should make little difference either at low optical depths (for which they are unimportant) or at high optical depth (for which memory of the quantitative form of the softening process is lost). This justifies our neglect of the 
process $\gamma+\gamma \rightarrow e^{+}+e^{-}+\gamma$, which is complementary (and comparable) to $e^{+} e^{-} \rightarrow \gamma+\gamma+\gamma$ which we do include.

\section{METHOD}

The nonlinear nature of the coupled radiative transfer equation (12) and the electron density equation (14) poses problems for their solution. Eleven spatial zones, logarithmically spaced in radius out to ten times $\mathrm{R}$, the inner radius, were employed. Numerical instability would occur in an explicit integration of the transport terms if the time step employed were such that a photon could traverse more than one spatial zone in one step (i.e., for $\Delta t \geq 0.259 R / c$ for the innermost zone); in order to improve the accuracy of the time-dependent solution, we used a substantially smaller $\Delta t=0.05 R / c$. A fully explicit treatment of the problem is impractical, except at low optical depths, because $\Delta t$ must also be chosen small enough that the interactions do not destroy all the photons (or all the charged particles) in a single step. At low optical depths, this condition is less stringent than that imposed by the transport terms. However, at high optical depths, the time steps permitted by an explicit solution become very small, because the particle and photon densities are large, and their interaction rates (per particle or photon) are proportional to their densities, and the computational effort increases rapidly.

In practice, we used a semi-implicit technique, in which the transport terms were evolved explicitly in time, while the local interaction terms were solved implicitly, by matrix inversion. This semi-implicit method is not unconditionally stable, but it is much more stable than explicit methods. In addition, the semi-implicit method required less memory than would be needed for a fully implicit technique. One factor which has been found to influence stability is the temporal dependence of the source term $S(E, r)$ : if a strong source is turned on suddenly, the semi-implicit method is made unstable by the

rapidly rising photon and particle densities. We therefore turned the source on gradually, with $S$ proportional to $1-\exp \left(-t / t_{0}\right)^{2}$, with $t_{0}=R / c$. The desired steady state solutions 
are obtained for $t \gg t_{0}$.

The semi-implicit method consists of the following: a transport equation

$$
\frac{\partial \psi}{\partial t}=G[r, t, v, E, \hat{\Omega}, \psi]-v \hat{\Omega} \cdot \nabla \psi
$$

is approximated by the difference equation

$$
\frac{\psi(t+\Delta t)-\psi(t)}{\Delta t}=G[r, t, v, E, \hat{\Omega}, \psi(t+\Delta t)]-v \hat{\Omega} \cdot \nabla \psi(t)
$$

with $\hat{\Omega} \cdot \nabla \psi$ approximated by the appropriate (upstream) explicit differencing scheme. The difference equation is linearized in the quantity $\Delta \psi=\psi(t+\Delta t)-\psi(t)$ and then solved for $\Delta \psi$ :

$$
\Delta \psi=\left[1-\frac{\partial G}{\partial \psi} \Delta t\right]^{-1}(G-v \hat{\Omega} \cdot \nabla \psi) \Delta t
$$

The field $\psi$ will in general depend on $E, \hat{\Omega}$, and $r$, as well as on time; as a result, the equation must be discretized in these other quantities as well. Write

$$
\psi_{j}^{i}=\psi\left(r=r^{i}, E=E_{j}, \hat{\Omega}=\hat{\Omega}_{j}, \ldots\right),
$$

with the index $i$ standing for purely positional information and $j$ and $k$ standing for everything else. Then define

$$
A_{j k}^{i} \equiv \delta_{j k}-\frac{\partial G_{j}^{i}}{\partial \psi_{k}^{i}}
$$

The $A_{j k}^{i}$ may be regarded as forming the components of a set of matrices $A^{i}$. Then

$$
\Delta \psi^{i}=\left(A^{i}\right)^{-1}\left(G^{i}-v \hat{\Omega} \cdot \nabla \psi^{i}\right) \Delta t
$$

In the present problem, $\psi$ refers to both the radiation intensity $I$ and the charged particle density $n_{e}$. The index $i$ labels the radial zones $\left(r=r_{i}\right)$, while the indices $j$ and $k$ refer to energy, direction, and species (photon or charged particle). The function $G$ includes an explicit source of photons as well as terms corresponding to pair production by photons and two- and three-photon pair annihilation. The spatial and angular derivative terms $\hat{\Omega} \cdot \psi$ 
were written solely in terms of $\psi(t)$; were these terms also linearized in $\Delta \psi$, one would arrive at a fully-implicit algorithm. For each radial zone $r_{i}$, the matrix $A^{i}$ is inverted, and $\Delta \psi^{i}$ is found; this process is repeated at each radial zone, and at each time-step, until convergence to a steady state is achieved.

The photon source spectrum was usually taken to have a power-law form in energy, and to fall off as $r^{-6}$. Such a spatial dependence might be expected if the burst were driven by magnetic reconnection, as is a Solar flare, because a dipole field's energy density has this dependence, or if the field's stress were to limit the confinement of the source's energy density. In general, models which require the diffusion of near-equilibrium radiation through an opaque atmosphere (a class which includes both thermonuclear and accretional impact models) naturally produce black body spectra with $T \sim 10^{7}{ }^{\circ} \mathrm{K}$, inconsistent with the much harder, probably nonthermal (Katz 1985) spectra of gamma-ray bursts. Nonthermal models usually require that the magnetic field produce the radiation or confine the radiating particles (Katz 1982), justifying our use of an $r^{-6}$ source dependence.

Eight energy and eight angular bins were employed. The angular bins were chosen to be those of the eight-point Gauss-Legendre quadrature scheme; the energy bins were spaced logarithmically.

\section{RESULTS}

Emergent steady-state spectra are shown in Figures 1-5; unless otherwise stated the source spectrum is of the form $S \propto E^{-\nu} r^{-6}$ and normalized by the parameter $\beta$ (Equation 11). Results for low values of $\beta$ are given in Figure 1 (for $\nu=1$ ) and in Figure 2 (for $\nu=2$ ). At very low source strengths (low $\beta$ ), the optical depth is small, and most photons escape the source region without interacting; the pair density is low, and the emergent spectrum is proportional to the source spectrum. At somewhat higher source rates, an apparent annihilation feature (either a peak or a broad "shelf" in the spectrum) is formed, and the high-energy portion of the spectrum becomes increasingly depleted. Results for 
$\nu=2$ and $\beta>1$ are shown in Figure 3. The depletion of high-energy photons increases as $\beta$ increases. One striking feature of the calculations is that the high-energy portion of the emergent spectrum is almost independent of $\beta$ in both shape and magnitude; as $\beta$ increases from 10 to $10^{4}$, the flux at $700 \mathrm{keV}$ increases by only a factor of about 10 , while the flux at $1 \mathrm{MeV}$ and above is essentially constant for $1<\beta<10^{4}$.

How sensitive is this result to the assumptions which underlie the calculations (specific interactions included, choice of the explicit source of photons, etc.)? In order to answer this question, calculations where performed with somewhat different assumptions. In one calculation, the charged-particle temperature was, at each time step, reduced to half of what it was calculated to be; this simulates the effect of charged particles radiating a significant fraction of their energy as low-energy photons (by synchrotron emission, for example). The result is shown in Figure 4, together with the result of the corresponding "standard" case $(\nu=2)$. In another calculation, the source spectrum was taken to be an unnormalized Wien spectrum corresponding to a photon temperature of $511 \mathrm{keV}$; for photon energies $<3 \mathrm{MeV}$, this distribution is much harder than the power-law $(\nu=2)$ spectrum used in the other computations. The results of this calculation, and of the corresponding "standard" case, are shown in Figure 5.

At high values of $\beta$ the high-energy portion is insensitive to the details of the source spectrum. This is illustrated in Figure 5: the emergent spectrum for a Wien-distribution source is essentially identical to that for a power-law source at energies above $300 \mathrm{keV}$.

Although the emergent spectrum is insensitive to the energy dependence of the source spectrum, it is quite sensitive to changes in the spatial dependence of the source. Photons produced near the star's surface have become well collimated by the time they have gone several star radii (this is a consequence of the spherical geometry; far from the source, all photons move nearly radially outward); any high-energy photons introduced far above the star's surface, and which are also travelling roughly radially outward, are below the kinematic threshold (Equation 1) to pair production. Therefore, the optical depth in a 
zone far above the star's surface is reduced below what it would have been in slab geometry, for which there is no such geometrical collimation effect. By contrast, a source confined to a thin shell at the surface can only produce photons in a region which is effectively slab-symmetric, so its pair-production optical depth is not reduced, and very few high energy photons emerge.

A source even more extended than our $r^{-6}$ source might be expected to yield a still larger high-energy flux. The investigation of such a source would require that the transport calculations be extended to larger distances (since the effective source radius grows as the source becomes more spatially extended). The short $(\sim 0.1 \mathrm{~ms})$ time-scales over which the intensities of some bursts have been observed to vary imply source regions no larger than $\sim 30 \mathrm{~km}$, excluding more extended source models, at least for these objects. In addition, the upper bound on high-energy flux at the source is inversely proportional to the source radius (because the optical depth scales roughly as $n_{\gamma} r_{e}^{2} R$, or, equivalently, because of the scale invariance of the transport equations). The observed flux is thus only linearly proportional to the source radius; the upper bound on source distance rises only as the square root of the source radius.

\section{CONCLUSIONS}

A comparison between the observed (initial) spectrum of the 5 March 1979 event and two model calculations is shown in Figure 6. For the purposes of comparison, the computed results are scaled to a neutron star $10 \mathrm{~km}$ in radius and $1 \mathrm{Kpc}$ from the Solar System. This distance is the largest for which the calculated emergent flux at $E>511 \mathrm{KeV}$ could even approximately fit the data. The low-energy portions of the spectra are in poor agreement with one another, but the low-energy emergent spectrum depends strongly on the source spectrum (and, in any case, it is the high-energy spectrum which has yielded upper bounds on the distance to the source of this burst). Above about $300 \mathrm{keV}$ (where wildly dissimilar sources yield almost identical fluxes), the calculated and observed spectra are in better 
agreement. This agreement suggests that the source of the 5 March 1979 event is no farther than about $1 \mathrm{kpc}$ from the Earth. A larger source region (either from a source which fell off more slowly than $r^{-6}$ or from a neutron star larger than $10 \mathrm{~km}$ in radius) could be somewhat farther away; however, because the distance limit scales only as the square root of the source radius, it cannot be relaxed significantly (a factor of 2 or 3 ) under any set of reasonable assumptions consistent with the observed $\leq 0.15 \mathrm{~ms}$ rise-time for that burst. Increasing the energy release rate (and hence, $\beta$ ) would not increase the high-energy flux (as is shown in Figure 3); increasing the hardness of the source spectrum will also not increase the flux significantly (as shown in Figure 5) for any "reasonable" source spectrum.

This distance limit is larger, by nearly an order of magnitude, than that given by Epstein (1985). In part, this difference results from Epstein's use of the condition $\tau<1$ to limit the high-energy flux, while we considered transport at arbitrary optical depths. The larger part of the discrepancy, however, is due to the difference in our assumed sources: Epstein considered a source limited to the surface of a neutron star, while we employed a source which extended above the surface. As mentioned previously, the use of a source limited to the star's surface (or to a thin shell around the star) results in a significantly smaller high-energy flux, in broad agreement with Epstein's work.

Are there any possible loopholes, ways in which the distance limit presented here could be evaded? Of the four possibilities listed in the Introduction:

1. Diffusion through optically thick regions is found to be ineffective.

2. Softening of the spectrum lets the radiant energy emerge (the required fluxes are far below the Planck function, justifying our neglect of stimulated emission), but does not relax a bound imposed by the observed high-energy flux. It does, however, explain the unusually soft spectrum observed for the very luminous March 5, 1979 event (assuming the LMC identification to be correct).

3. Our calculations show that the suggested "window" in the pair production opacity is 
not effectively used by the flux; it does not self-collimate.

4. The sudden release of energy in the form of gamma-rays and relativistic particles, even if initially uncollimated, will, if unconfined, lead to relativistic outward streaming motion and an outwardly collimated radiation field. The kinematic threshold to pair production (Equation 1) would then suppress the pair production rate and the optical depth, permitting the escape of multi-MeV photons. Values of magnetic fields characteristic of pulsars would interfere with radial outflow, but a fireball of particles and photons produced near the surface could oscillate between magnetic hemispheres, and during its upward motion produce collimated outward flow. Alternatively, particle production may occur on the narrow funnel of open field lines near the magnetic pole of a high field neutron star; this funnel may be widened if sufficient energy is released to distort the magnetic field configuration and "blow out" part of the magnetosphere.

Another way to evade the distance limit is based on the pulse pile-up effect suggested by Laros et al. (1985): a gamma-ray scintillation detector will count two (or more) photons as one photon (with energy equal to the total energy of all the photons) if they are received within a short time interval (of the order of a microsecond). If the energy of a burst were concentrated in a series of short "micro-bursts", rather than being more or less smoothly varying with time, it may be possible to account for most (or all) the observed high-energy flux in terms of micro-bursts of soft photons. The plausibility of this explanation rests, however, on the response times of detector scintillators and on the minimum variability time-scales of GRBs. From light travel time arguments, the latter quantity cannot be much less than about 30 microseconds if the burst source is the whole surface of a neutron star, while even for radiation from a small $(\sim 1 \mathrm{~km})$ region on a neutron star, the limit is about 3 microseconds. Pulse pile-up may, but need not, be important if GRBs involve hot spots on neutron-star surfaces; if the source region covers the entire surface of a neutron star, however, pile-up is probably unimportant. In any case, theorists ought to be reluctant to claim the observations have been misinterpreted (as would be the case if pile-up were 
significant) without strong evidence.

It is therefore not possible to resolve definitively the apparent conflict between the statistically attractive identification of the March 5, 1979 gamma-ray burst with N49 in the LMC and the pair production bound on its MeV gamma-ray flux. Relativistic outflow and collimation may be the most attractive hypothesis, for they would also resolve the problem (Imamura and Epstein 1987) of the near absence of reprocessed X-rays from the neutron star's surface. Alternatively, the paucity of reprocessed X-rays may be explained as a consequence of self-absorption, if gamma-ray burst distances are $\sim 100 \mathrm{Kpc}$, as may be implied by data from the BATSE on GRO (Katz 1992). Pulse pileup or an accidental positional coincidence cannot be excluded.

Observations of MeV gamma-rays from other bursters (Matz, et al. 1985) raise similar issues, especially if their sources are very distant. Because gamma-ray bursts are diverse, problems and solutions found in one class of them (such as soft gamma repeaters like the March 5, 1979 event) need not be applicable to all.

Throughout this paper we have assumed that the charged particles are confined by a strong magnetic field. Consequently, annihilation gamma-rays are produced with zero mean momentum (which we approximate as an isotropic source). This constrains their transport, and from it follow the limits on the escaping hard photon flux and on the inferred distances to gamma-ray burst sources.

The GRO observed (Meegan, et al. 1992) the sources of gamma-ray bursts to be isotropically distributed on the sky; when combined with the distribution of burst fluences this leads to the inference that the sources are distributed either in a very extended Galactic halo or at cosmological distances. At the luminosities implied by halo distances a neutron star's magnetic field may not be sufficient to confine the pair gas; at cosmological luminosities expected neutron star magnetic stresses are insignificant. An unconfined pair gas should therefore be considered.

An optically thick mixture of gas and radiation may be considered as a single fluid 
with a combined equation of state; it is not necessary to consider radiation transport in order to describe its flow. At semi-relativistic temperature the sound speed $c_{s}$ approaches $c / 3^{1 / 2}$. If produced impulsively the opaque fluid undergoes nearly adiabatic expansion, cooling on a time scale $\sim r / c_{s}$. In the course of this expansion the thermal energy, residing in an isotropic distribution of photon and charged particle momenta, is converted to radially collimated motion whose mean velocity is at least semi-relativistic. The pairs, now adiabatically cooled to $k_{B} T \ll m_{e} c^{2}$, annihilate. The photons are described by the same low temperature $T$ in the fluid frame and are below the pair production threshold; in the laboratory frame they are collimated in the outward direction and may have multi-MeV energies.

The emergent spectrum depends on details of the equilibration process. In the simplest model photons and pairs are initially in equilibrium at temperature $T_{0}$ (at optical depths $<\alpha^{-1}$ this may not be the case, and the photon chemical potential may be negative rather than zero). Adiabatic expansion only scales down the photon energy and temperature in the distribution function, but does not change the spectral shape; the Doppler shift to the observer's frame scales them up again. The total photon number and energy are conserved in adiabatic expansion of a photon gas, so the emergent spectrum (in the observer's frame) would be identical to that of the initial black body. Annihilation of the pairs increases the photon temperature by a factor between 1 (for $k_{B} T_{0} \ll m_{e} c^{2}$ ) and $(11 / 4)^{1 / 3}$ (for $\left.k_{B} T_{0} \gg m_{e} c^{2}\right)$. Power law spectral tails cannot be explained by these processes, and require departures from equilibrium. For example, nonequilibrium survival of pairs in the rarefying and cooling outflow (requiring low densities) may lead to a high energy annihilation spectrum with an abrupt cutoff at $2 \gamma m_{e} c^{2}$, where the usual Lorentz factor $\gamma$ is obtained from the bulk expansion speed. The value of $\gamma$ depends on $T_{0} ; \gamma \sim k_{B} T_{0} / m_{e} c^{2}$ if $k_{B} T_{0} \gg m_{e} c^{2}$ and $\gamma \gg 1$ if the pair density is very low $\left(k_{B} T_{0} \ll m_{e} c^{2}\right.$ in equilibrium), while an equilibrium gas with $k_{B} T_{0} \sim m_{e} c^{2}$ leads to $\gamma \sim O(1)$.

The expanding pair-photon plasma is optically thick, and does not become thin until 
its radius $r \sim\left(\sigma N_{p}\right)^{1 / 2}$, where $N_{p}$ is the total number of photons and particles impulsively produced (for steady energy release use $N_{p} \sim \dot{N} r / c$ to obtain $r \sim \sigma \dot{N} / c$; the smaller of these two estimates of $r$ is applicable). Substitution of $\sigma_{e s} \sim r_{e}^{2}$ leads to very large values of $r\left(\sim 10^{13} \mathrm{~cm}\right.$ for Galactic halo bursts and $\sim 10^{16} \mathrm{~cm}$ for cosmological bursts $)$. These values are deceptive - once the fluid-frame temperature (varying $\propto r^{-1}$ in the adiabatic expansion of a relativistic fluid) drops to values $\ll m_{e} c^{2}$ the photons fall below the pair-production threshold and the pairs rapidly annihilate and disappear. Although the column density of photons far exceeds $\sigma_{e s}^{-1}$ they stream outward freely, and the effective photosphere forms at $r \sim r_{0}\left(k_{B} T_{0} / m_{e} c^{2}\right)$. The observed pulse of radiation has a duration $\sim r /\left(\gamma^{2} c\right) \sim$ $r_{0} m_{e} c /\left(k_{B} T_{0}\right) \sim 10^{-4}$ sec because of its angular collimation to a width $\theta \sim 1 / \gamma$. It is clear that models of this kind cannot explain the observed durations and complex timehistories of gamma-ray bursts unless there is a continuing release of energy throughout the observed burst duration, in the range $10^{-1}-10^{3}$ seconds. Such a continued release is plausible in "solar-flare" models of bursts (Ruderman 1975, Katz 1982) but may be difficult to accomodate in catastrophic events (neutron star birth, coalescence, or death) at cosmological distances.

The inference of Galactic halo distances $\sim 100 \mathrm{Kpc}$ to gamma-ray bursts (Meegan, et al. 1992; Katz 1992) answers one of the chief objections to magnetic flare models. They have suffered from the difficulty of tapping the magnetostatic energy of a magnetosphere whose source currents are in the neutron star's interior. Magnetic reconnection, the likely power supply to a flare, cannot occur in usual models of neutron star magnetospheres in which current flows only on open field lines. Flares can occur if current loops from the interior erupt into the magnetosphere. This occurs readily in ordinary fluid stellar atmospheres. In neutron stars it requires magnetic stresses sufficient to break the solid crust, whose strength $\mu_{0}$ is uncertain but may be $\sim 10^{25}$ dyne $/ \mathrm{cm}^{2}$ in the outer crust and $\sim 10^{27}$ dyne $/ \mathrm{cm}^{2}$ in the inner crust (Ruderman 1972). A magnetic stress of this value implies a characteristic flare energy $\sim \mu_{0} \ell^{3} \sim 10^{40}-10^{42} \mathrm{erg}$, where $\ell \sim 10^{5} \mathrm{~cm}$ is 
the crust's thickness. This crude estimate is consistent with halo distances (but not with Galactic disc distances $\sim 100 \mathrm{pc}$ formerly considered likely). The narrow range of magnetic fields inferred (Murakami, et al. 1988; Fenimore, et al. 1988) from spectral data is also explained if the magnetic stress in a burst is determined by the crustal strengths found in a homogeneous population of neutron stars. The deficiency of weak bursts observed by GRO implies that the energy release is (broadly) concentrated in events around the knee of the $\log N / \log S$ curve, which corresponds to $\sim 10^{43}$ erg at $100 \mathrm{Kpc}$ distance.

We thank F. Perkins and M. A. Ruderman for discussions and NASA grants NAGW592 and NAGW-2028 for support. 


\section{References}

Carrigan, B. J. 1987, Ph. D. Thesis, Washington U., St. Louis.

Carrigan, B. J., and Katz, J. I. 1984, Astron. Exp. 1, 89.

Carrigan, B. J., and Katz, J. I. 1987, Ap. J. 323, 557.

Cavallo, G., and Rees, M. J. 1978, M. N. R. A. S. 183, 359.

Cline, T. L. 1980, Comments Ap. 9, 13.

Epstein, R. I. 1985, Ap. J. 297, 555.

Fenimore, E. E., Laros, J. G., Klebesadel, R. W., Stockdale, R. E., and Kane, S. R. 1982, in Gamma Ray Transients and Related Astrophysical Phenomena, eds. R. E. Lingenfelter, H. S. Hudson, D. M. Worrall (New York: AIP) p. 201.

Fenimore, E. E., et al. 1988, Ap. J. (Lett.) 335, L71.

Guilbert, P. W., and Stepney, S. 1985, M. N. R. A. S. 212, 523.

Helfand, D. J., and Long, K. S. 1979, Nature 282, 589.

Imamura, J. N., and Epstein, R. I. 1987, Ap. J. 313, 711.

Katz, J. I. 1982, Ap. J. 260, 371.

Katz, J. I. 1985, Ap. Lett. 24, 183.

Katz, J. I. 1987, High Energy Astrophysics (Menlo Park: Addison-Wesley).

Katz, J. I. 1992, Ap. Sp. Sci. in press.

Laros, J. G., Fenimore, E. E., Fikani, M. M., Klebesadel, R. W., van der Klis, M., and Gottwald, M. 1985, Nature 318, 448.

Liang, E. P., and Petrosian, V., eds. 1986, Gamma-Ray Bursts (New York: AIP). 
Matteson, J. L. 1983, in Positron-Electron Pairs in Astrophysics, eds. M. L. Burns, A. K. Harding, R. Ramaty (New York: AIP) p. 292.

Matz, S. M., Forrest, D. J., Vestrand, W. T., Chupp, E. L., Share, G. H., and Rieger, E. 1985, Ap. J. (Lett.) 288, L37.

Meegan, C. A., et al. 1992, Nature 355, 143.

Murakami, T., et al. 1988, Nature 335, 234.

Nolan, P. L., Share, G. H., Chupp, E. L., Forrest, D. J., and Matz, S. M. 1984, Nature 311, 360 .

Ruderman, M. A. 1972, Ann. Rev. Astr. Ap. 10, 427.

Ruderman, M. A. 1975, Ann. N. Y. Acad. Sci. 262, 164.

Schmidt, W. K. H. 1978, Nature 271, 525.

Svensson, R. 1983, Ap. J. 270, 300.

Teegarden, B. J. 1984, in High Energy Transients in Astrophysics, ed. S. E. Woosley (New York: AIP) p. 352. 


\section{Figure Captions}

Figure 1: Emergent spectra (in arbitrary units) for $\nu=1$. Dashed-dotted curve: $\beta=0.01$. Dashed curve: $\beta=0.03$. Dotted curve: $\beta=0.1$. Solid curve: $\beta=0.3$.

Figure 2: Emergent spectra for $\nu=2$. Solid curve: $\beta=0.03$. Dashed-dotted curve: $\beta=0.1$. Dashed curve: $\beta=0.3$. Dotted curve: $\beta=1$.

Figure 3: Emergent spectra for $\nu=2$. Solid curve: $\beta=10$. Dashed-dotted curve: $\beta=100$. Dashed curve: $\beta=10^{3}$. Dotted curve: $\beta=10^{4}$.

Figure 4: Emergent spectra with and without additional cooling of the electron-positron gas. $\beta=10, \nu=2$. Dotted curve: no additional cooling. Dashed curve: electron-positron temperature reduced to half its nominal value.

Figure 5: Emergent spectra for $\beta=10^{4}$. Dotted curve: Source $\propto E^{-2}(\nu=2)$. Dashed curve: Source $\propto E^{2} \exp (-E)$ (Wien-like source spectrum).

Figure 6: Comparison of calculated flux (photons $\mathrm{cm}^{-2} \mathrm{~s}^{-1} \mathrm{keV}^{-1}$ ) for $\nu=2, \beta=10^{4}$ (dotted line) and $\beta=10^{3}$ (dashed line) to the spectrum of the initial 4 seconds of the March 5, 1979 burst. The calculated fluxes assumed a $10 \mathrm{~km}$ radius neutron star $1 \mathrm{Kpc}$ from the solar system. 